\title{
Inductance and resistance calculations for a pair of rectangular conductors
}

\author{
K.F. Goddard, A.A. Roy and J.K. Sykulski
}

\begin{abstract}
Various semi-analytical and numerical calculations to compute the inductance and resistance of a pair of rectangular conductors are reviewed. The DC inductance of infinitely thin strips and strips of finite thickness are specifically considered. In the former case, the inductance is computed using the $T-\Omega$ method, whereas in the latter case it is computed by direct integration using Maple. In both cases, the results are checked using finite element analysis. It is also shown how the DC inductance of three strips may be computed using the method of superposition of solutions. Conformal mapping theory is used to obtain the inductance of a pair of infinitely conducting strips. Infinitely thin strips and strips of finite thickness are considered and the inductance is compared against results obtained using finite element analysis. A method of estimating the resistance of the strips is described which is expected to give useful results when the skin depth is small in relation to the thickness of the strips.
\end{abstract}

\section{DC inductance of a pair of infinitely thin strip conductors}

When the current distribution is known, the inductance can be obtained using the $T-\Omega$ method. The field is modelled as the sum of two components such that $\boldsymbol{H}=\boldsymbol{T}+\nabla \Omega$. The vector field $\boldsymbol{T}$ must satisfy $\nabla \times \boldsymbol{T}=\boldsymbol{J}$. For the geometry shown in Fig. 1, this is most easily achieved by setting $T_{x}=I / w$ in the space between the conductors and zero elsewhere.

The scalar potential field $\Omega$ is defined to ensure that $\nabla \cdot \boldsymbol{B}=0$. When the permeability is uniform, the field $\nabla \Omega$ is obtained by integrating the field of magnetic polarity sources located where $\nabla . T \neq 0$. These fields are described in Fig. 2.

For any $T-\Omega$ solution, the inductive energy is given by

$$
\frac{1}{2} I^{2} L=\frac{1}{2} \iint \boldsymbol{T} \cdot \boldsymbol{B} d v
$$

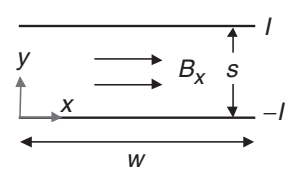

Fig. 1 Pair of conducting strips carrying currents $I$ and $-I$

The inductance per unit length is obtained by integrating $B_{x}$ over the rectangular area between the strips and dividing by

(C) IEE, 2005 (contents include material subject to (C) Crown Copyright 2004 Dstl)

IEE Proceedings online no. 20041058

doi:10.1049/ip-smt:20041058

Paper received 25th March 2004

The authors are with the School of Electronics and Computer Science, University of Southampton, Southampton, UK

E-mail: J.K.Sykulski@soton.ac.uk

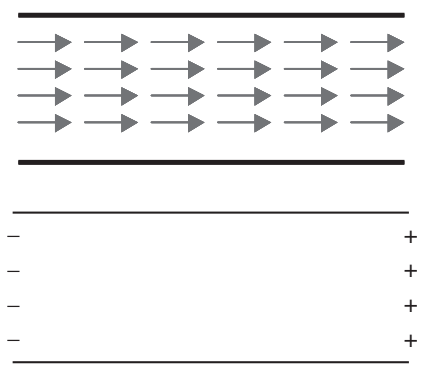

Fig. 2 The vector field $\boldsymbol{T}$ and the sources of the conservative field $\nabla \Omega$

$w I$. The flux due to the vector field $\boldsymbol{T}$ is $\mu_{0} I s / w$ but there is a negative contribution from the field $\nabla \Omega$.

The inductance per unit length $(L)$ is then given by:

$$
\begin{aligned}
L & =\frac{1}{w I} \int_{x=0}^{w} \int_{y=0}^{s}\left(\mu_{0} T_{x}-4 \int_{h=0}^{y} \frac{\mu_{0} T_{x}}{2 \pi} \frac{x}{x^{2}+h^{2}} d h\right) d y d x \\
& =\frac{\mu_{0} s}{w}-\frac{2 \mu_{0}}{w^{2} \pi} \int_{x=0}^{w} \int_{y=0}^{s} \tan ^{-1}\left(\frac{y}{x}\right) d y d x
\end{aligned}
$$

Evaluating the integrals we obtain:

$$
\begin{aligned}
L & =\frac{\mu_{0} s}{w}-\frac{2 \mu_{0}}{\pi} \\
& \times\left\{\frac{s^{2} \ln \left(1+(w / s)^{2}\right)}{4 w^{2}}+\frac{s \tan ^{-1}(s / w)}{w}-\frac{\ln \left(1+(s / w)^{2}\right)}{4}\right\}
\end{aligned}
$$

In the limit when $s / w$ is large (1) becomes:

$$
L=\frac{\mu_{0}}{\pi}\left(\ln \frac{s}{w}+\frac{3}{2}\right)
$$

Figure 3 shows that when $s>w$ a reasonable approximation is obtained using the simpler formula. Large separation 


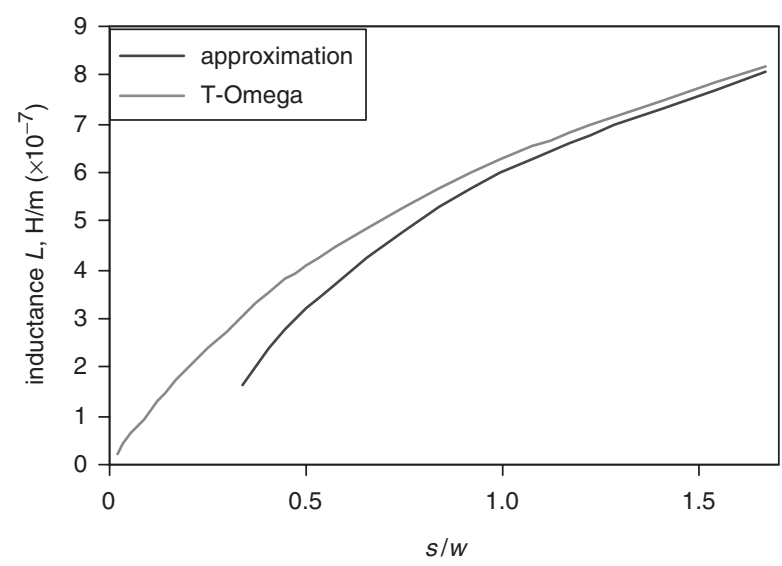

Fig. 3 Comparison of inductance calculated using the $T-\Omega$ method with the large separation approximation

approximations are also available for conductors of other shapes and a range of frequencies [1].

\section{DC inductance of a pair of rectangular conductors}

The DC inductance of a pair of rectangular conductors of finite thickness may be obtained as follows. In general the vector potential at any point $(u, v)$ is given by:

$$
A_{z}=-\frac{\mu_{0}}{2 \pi} \iint \frac{1}{2} \ln \left((x-u)^{2}+(y-v)^{2}\right) J_{z}(x, y) d x d y
$$

and the inductance is given by $L=\iint \boldsymbol{A} . \boldsymbol{J} d u d v / I^{2}$. The total inductance of two conductors in series is given by

$$
L=L_{1}+L_{2}+2 M
$$

where $L_{1}$ and $L_{2}$ are the self-inductances of the two conductors and $M$ is the mutual inductance between them.

Since $\boldsymbol{A}$ is obtained from a double integral, $L$ is given by a quadruple integral. For the self-inductance of a rectangular conductor of dimensions $w$ and $t$, this quadruple integral can be reduced to a double integral using:

$$
\int_{0}^{L} \int_{0}^{L} f(|s-t|) d s d t=2 \int_{0}^{L}(L-u) f(u) d u
$$

where $u=|s-t|$. Hence, the self-inductance is given by

$$
L_{1}=L_{2}=-\frac{\mu_{0}}{2 \pi} 4 \int_{0}^{w} \frac{w-p}{w^{2}} \int_{0}^{t} \frac{t-q}{t^{2}} \frac{1}{2} \ln \left(q^{2}+p^{2}\right) d q d p
$$

where $p=|x-u|$ and $q=|y-v|$.

The calculation for mutual inductance $M$ has less symmetry; hence, $M$ is given by the sum of two double integrals. For the simple geometry in Fig. 4, $M$ is given by

$$
\begin{aligned}
M= & \frac{\mu_{0}}{2 \pi} 2 \int_{0}^{w} \frac{w-p}{w^{2}} \int_{0}^{t} \frac{t-q}{t^{2}}\left(\frac{1}{2} \ln \left((s+q)^{2}+p^{2}\right)\right. \\
& \left.+\frac{1}{2} \ln \left((s-q)^{2}+p^{2}\right)\right) d q d p
\end{aligned}
$$

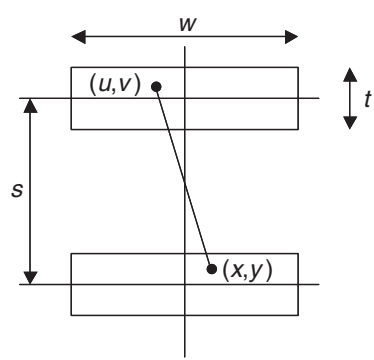

Fig. 4 Position of points in two rectangular conductors

where $p=|x-u|$ and $s \pm q=|y-v|$. The integrals for $L_{1}$ and $M$ were evaluated using Maple and combine to give:

$$
\begin{aligned}
L= & \left(\mu_{0} /\left(24 \pi w^{2} t^{2}\right)\right)\left[16\left(t^{3} w-w^{3} t\right) \tan ^{-1}(t / w)\right. \\
& -8 \pi t^{3} w+16\left(w^{3} s-s^{3} w\right) \tan ^{-1}(w / s) \\
& -\left((s+t)^{4}-6 w^{2}(s+t)^{2}+w^{4}\right) \ln \left((s+t)^{2}+w^{2}\right) \\
& -\left((s-t)^{4}-6 w^{2}(s-t)^{2}+w^{4}\right) \ln \left((s-t)^{2}+w^{2}\right) \\
& +8\left(w(s+t)^{3}-w^{3}(s+t)\right) \tan ^{-1}(w /(s+t)) \\
& +8\left(w(s-t)^{3}-w^{3}(s-t)\right) \tan ^{-1}(w /(s-t)) \\
& +2(s-t)^{4} \ln (s-t)+2(s+t)^{4} \ln (s+t)-4 t^{4} \ln (t) \\
& -4 s^{4} \ln (s)-4 w^{4} \ln (w) \\
& +2\left(w^{4}-6 w^{2} t^{2}+t^{4}\right) \ln \left(t^{2}+w^{2}\right) \\
& \left.+2\left(w^{4}-6 w^{2} s^{2}+s^{4}\right) \ln \left(s^{2}+w^{2}\right)\right] .
\end{aligned}
$$

Figure 5 shows the DC inductance against $s / w$ for various ratios of $t / w$. Also shown on this graph is the inductance obtained from (1) for infinitely thin strips. The similar shape of the graphs suggests that the inductance may be expressed in relation to (1). Indeed, when the formula is expanded in terms of $t / w$ we obtain:

$$
\begin{aligned}
L= & L(t=0)-\frac{\mu_{0} t}{3 w} \\
& +\frac{\mu_{0}}{24 \pi}\left(2 \ln \left(\frac{w^{2} s^{2}}{t^{2}\left(w^{2}+s^{2}\right)}\right)+\frac{25}{3}\right)\left(\frac{t}{w}\right)^{2} \\
& +O\left(\frac{t}{w}\right)^{4}
\end{aligned}
$$

For the range of data plotted in Fig. 5, this expression agrees to within $0.3 \%$ of the exact formula given in (2).

The exact formula was checked against a series of finite element models and found to agree to four significant figures. This result gives a high degree of confidence that the analytical formula is correct.

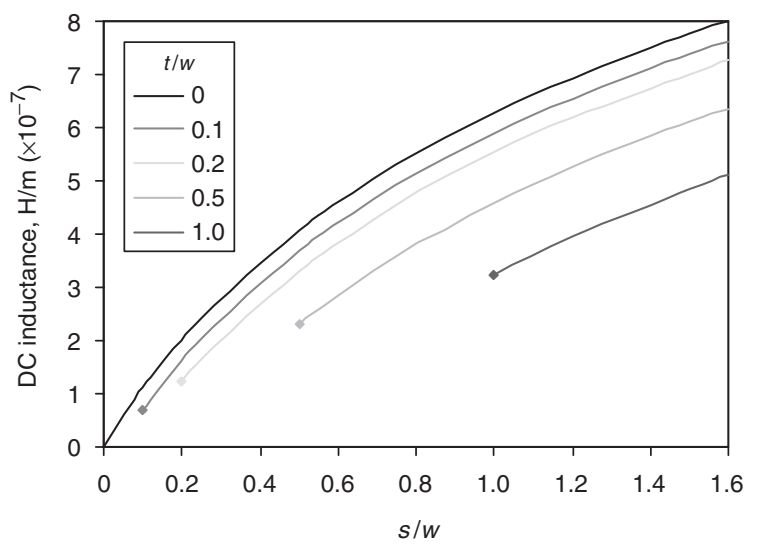

Fig. 5 The DC inductance for various $w$, $s$ and $t$ 


\section{DC inductance of a system of three strips}

The DC inductance of the three-strip configuration in Fig. 6 is obtained as follows.

The magnetic field may be obtained from the superposition of two simpler fields, as illustrated in Fig. 7. The contribution of each of those fields to the inductance may be obtained from the earlier analysis.

The total inductance is given by:

$$
L_{3}(s, w)=L(s, w)-\frac{1}{4} L(2 s, w)
$$

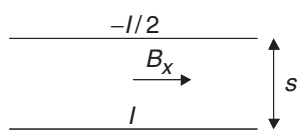

$\stackrel{-1 / 2}{\longleftarrow}$

Fig. 6 Three conducting strips

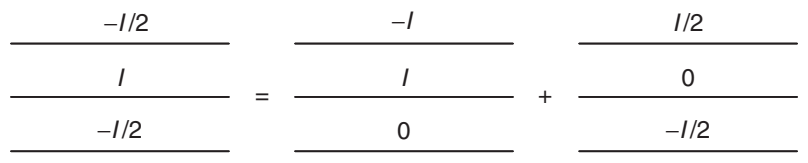

Fig. 7 Use of superposition to obtain the inductance of a threestrip circuit

where $L_{3}$ is the inductance for the three strips and $L$ is the inductance for the two strips, using (1).

An expression for the DC inductance of three strips of finite thickness may be found by using values of $L$ from (2) or (3). In this case, the total inductance is given by:

$$
L_{3}(s, w, t)=L(s, w, t)-\frac{1}{4} L(2 s, w, t)
$$

\section{Inductance of a pair of infinitely thin, infinitely conducting strips}

This calculation was performed using the composition of two conformal mappings. The first of these is the Jacobian elliptic function, which maps a rectangle in complex $z$-space, for which the inductance is easily calculated, onto the upper half plane as indicated in Fig. 8. This function, denoted by $w=\operatorname{sn}(z, k)$, is defined as the inverse of the function elliptic $F(w, k)$ [2]. The function has the following properties:

$\operatorname{sn}(K, k)=1, \operatorname{sn}\left(K+i K^{\prime}, k\right)=1 / k, \operatorname{sn}(-K, k)=-1$, and $\operatorname{sn}\left(-K+i K^{\prime}, k\right)=-1 / k$ where $K$ and $K^{\prime}$ are functions of $k$.

Figure 9 shows the mapping of the rectangle for $k=0.5$.

The mapping $z=F(w)=\operatorname{elliptic} F(w, k)$, which may be derived from the Schwartz-Christoffel transformation [2],
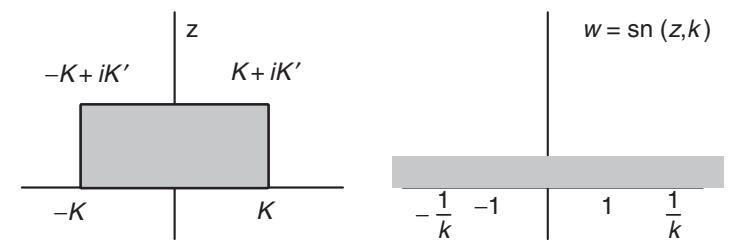

Fig. 8 The mapping $s n(z, k)$

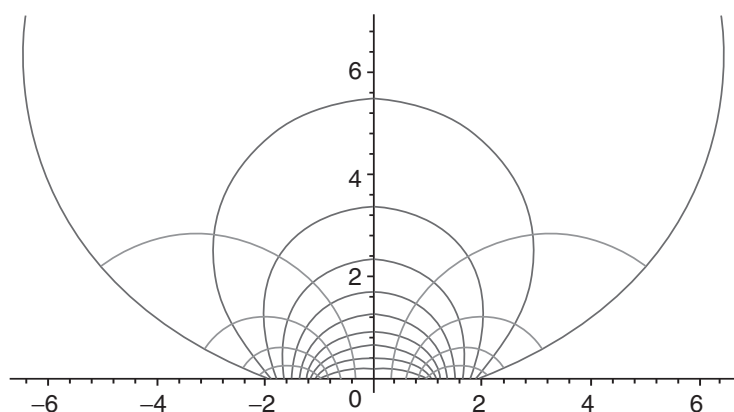

Fig. 9 Contours of vector and scalar magnetic potential under the map sn $z$

is given by:

$$
z=F(w)=\int_{0}^{w} \frac{d v}{\sqrt{\left(1-v^{2}\right)\left(1-k^{2} v^{2}\right)}}
$$

Having mapped the sides of the rectangle to the real axis it now remains to map the real axis in $w$-space to the geometry matching the two strips in $s$-space. This mapping, denoted by $s=p(w)$, is also found from the SchwartzChristoffel transformation and is given by:

$$
s=p(w)=\int_{0}^{w} \frac{(t+b)(t-b) d t}{\sqrt{(t+a)(t+c)(t-c)(t-a)}}
$$

Rearranging the integral we find that:

$$
p(w)=\left(c-\frac{b^{2}}{c}\right) \operatorname{elliptic} F\left(\frac{w}{a}, \frac{a}{c}\right)-\operatorname{celliptic} E\left(\frac{w}{a}, \frac{a}{c}\right)
$$

where

$$
\text { elliptic } E(w, k)=\int_{0}^{w} \sqrt{\frac{1-k^{2} t^{2}}{1-t^{2}}} d t
$$

The constants $a, b$ and $c$ refer to points on the real axis in $w$-space; point $b$ is mapped to the corner of the strip whereas points $a$ and $c$ are both mapped to a point midway along the side of the strip. The mapping is illustrated schematically in Fig. 10.

To ensure that, when $w=\operatorname{sn}(z, k)$, the conducting surfaces map to two opposite sides of the rectangle, we must set $c=1$ and $a=1 / k$. The condition $p(a)=p(1)$ means that $b$ may be expressed as a function of $a$. We find that:

$$
\begin{aligned}
b^{2}= & {\left[a^{2}(-\operatorname{elliptic} K(1 / a)-\operatorname{elliptic} E(a, 1 / a)\right.} \\
+ & \operatorname{elliptic} E(1,1 / a)+\operatorname{elliptic} F(a, 1 / a))] / \\
& {[\operatorname{elliptic} F(a, 1 / a)-\operatorname{elliptic} K(1 / a)] }
\end{aligned}
$$

Since $a$ and $b$ are functions of $k$, the mapping $s=p(\operatorname{sn}(z, k))$ can be calculated for any $k$. Figure 11 illustrates this mapping for $k=0.1$.
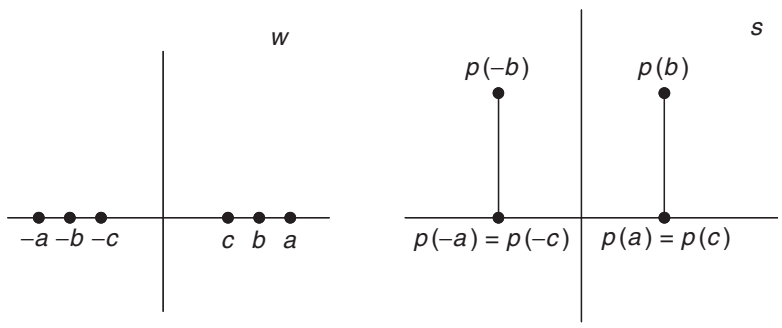

Fig. 10 The mapping $p(w)$ 


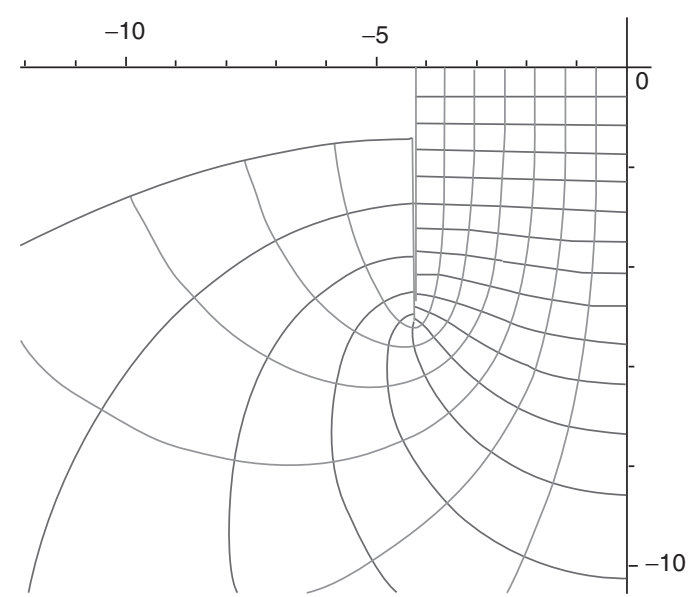

Fig. 11 Typical map showing contours of vector potential and scalar magnetic potential. Only one-quarter of the model is shown here

The ratio of separation to width in s-space is given by:

$$
\text { sep } / \text { width }=\Re(p(b)) / \mathfrak{T}(p(b))
$$

The value of $k$ that satisfies (4) was found using the secant algorithm. The inductance is then found from the ratio of the side lengths $K$ and $K^{\prime}$ of the rectangle, which are found from $K=F(1)$ and $i K^{\prime}=F(1 / k)-F(1)$.

Since elliptic $K(k)$ is defined by:

$$
\operatorname{elliptic} K(k)=\int_{0}^{1} \frac{d v}{\sqrt{\left(1-v^{2}\right)\left(1-k^{2} v^{2}\right)}}
$$

it is clear that $K=$ elliptic $K(k)$ and

$$
F(1 / k)=\frac{1}{k} \operatorname{elliptic} K\left(\frac{1}{k}\right) .
$$

It can be shown [3] that, for $|k|<1$ :

$$
\frac{1}{k} \operatorname{elliptic} K\left(\frac{1}{k}\right)=\operatorname{elliptic} K(k)-i \operatorname{elliptic} K\left(k^{\prime}\right)
$$

where $k^{\prime}=\sqrt{1-k^{2}}$. Hence, the inductance is given by:

$$
L=\mu_{0} \frac{K}{K^{\prime}}=\mu_{0} \frac{\operatorname{elliptic} K(k)}{\operatorname{elliptic} K\left(k^{\prime}\right)}
$$

A method of evaluating elliptic $K(k)$ for $|k|<1$ is given in [4], and described as follows. We begin with the triplet of numbers:

$$
l_{0}=1, \quad m_{0}=\sqrt{1-k^{2}}, \quad n_{0}=k
$$

Successive iterations of $l_{i}, m_{i}$ and $n_{i}$ are found by taking:

$$
\begin{aligned}
& l_{i}=\frac{1}{2}\left(l_{i-1}+m_{i-1}\right) \quad m_{i}=\sqrt{l_{i-1} m_{i-1}} \\
& n_{i}=\frac{1}{2}\left(l_{i-1}-m_{i-1}\right)
\end{aligned}
$$

Convergence is achieved when $n_{i} \approx 0$ and

$$
\text { elliptic } K(k)=\frac{\pi}{2 l_{i}}
$$

\subsection{Results}

The inductance, obtained from (5) is shown in Fig. 12, and compared with the DC inductance obtained from (1). For large separations the difference between these two inductance values tends to a constant value of $\mu_{0}(1.5-\ln (4)) / \pi$.

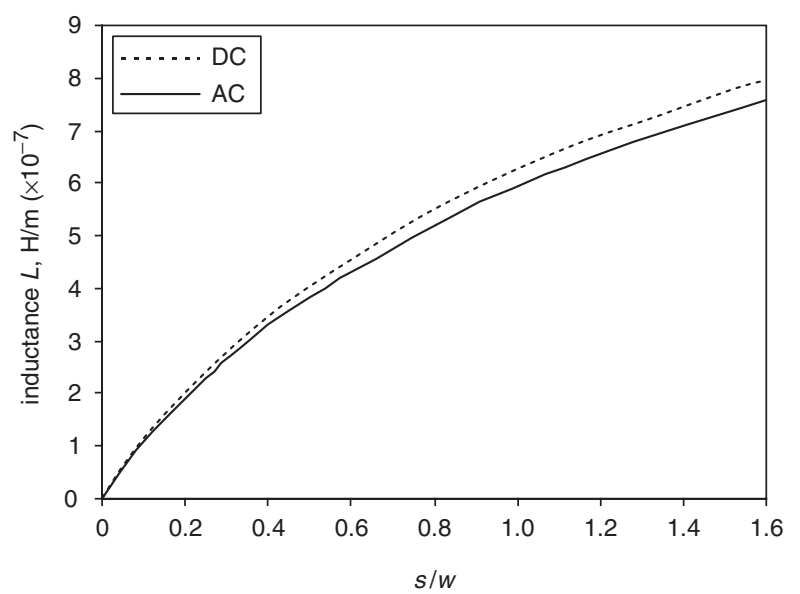

Fig. 12 Inductance as a function of separation/width

The high-frequency inductance estimates obtained using the above semi-analytical method were compared with values obtained using finite element analysis and found to agree to within $0.2 \%$. These finite element models use boundary conditions to represent the conductors. A fixed value of flux is imposed between the go and return conductors and the inductance per unit length is calculated using:

$$
L=\phi^{2} / \int \boldsymbol{B} . \boldsymbol{H} d A
$$

\section{Inductance of infinitely conducting strips of finite thickness}

When the strips have a finite thickness, the mapping $p(w)$ is more complicated, since the point $b$ at the edge of the strip has been replaced by a pair of points $b_{1}$ and $b_{2}$. The mapping, which is illustrated in Fig. 13, is given by the integral:

$$
s=p(w)=\int \frac{\sqrt{w+b_{1}} \sqrt{w+b_{2}} \sqrt{w-b_{2}} \sqrt{w-b_{1}}}{\sqrt{w+a} \sqrt{w+c} \sqrt{w-c} \sqrt{w-a}} d w
$$
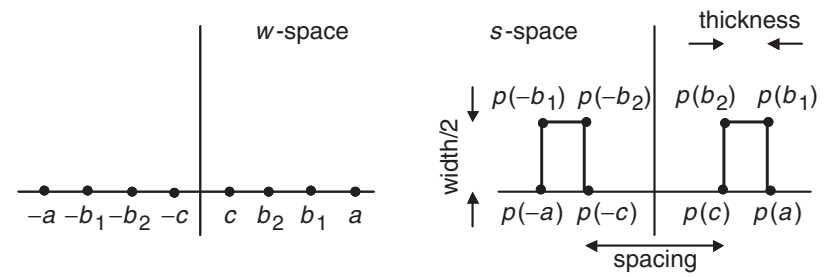

Fig. 13 The Schwartz-Christoffel transformation for the case of strips of finite thickness

Unfortunately, due to the complicated nature of this integral, we have been unable to find an explicit expression for $p(w)$. The integration between consecutive points on the real axis can, however, be performed numerically, making appropriate substitutions to remove the singularities in the denominator.

Suppose, for example, we wish to compute:

$$
\begin{aligned}
p\left(b_{2}\right)-p(c) & =\int_{c}^{b_{2}} \sqrt{\frac{\left(w^{2}-b_{1}^{2}\right)\left(w^{2}-b_{2}^{2}\right)}{\left(w^{2}-a^{2}\right)\left(w^{2}-c^{2}\right)}} d w \\
& =\int_{c}^{b_{2}} g(w) \sqrt{\frac{w-b_{2}}{w-c}} d w
\end{aligned}
$$


where

$$
g(w)=\sqrt{\frac{\left(w^{2}-b_{1}^{2}\right)\left(w+b_{2}\right)}{\left(w^{2}-a^{2}\right)(w+c)}}
$$

The singularities at the ends of the integration range may be removed using the substitution:

$$
w=\frac{c+b_{2}}{2}-\left(\frac{b_{2}-c}{2}\right) \cos \theta
$$

This gives

$$
p\left(b_{2}\right)-p(c)=i\left(b_{2}-c\right) \int_{0}^{\pi} g(w) \cos ^{2}\left(\frac{\theta}{2}\right) d \theta
$$

Numerical integration with respect to $\theta$ is now straightforward. The other integrals were transformed using similar substitutions. A 'black box' function was constructed to compute the image in $s$-space of the input vector $\left(a, b_{2}, b_{1}\right.$, $c$ ) in $w$-space. This function is used by a Matlab optimisation function to find the values of $a, b_{2}, b_{1}$ and $c$ which give rise to the given geometry. Having obtained the values of $a$ and $c$, the inductance is then found using (5) with $k=c / a$.

\subsection{Results}

The above method was used to estimate the inductance for a range of values of width, separation and thickness. These results were compared with those obtained from finite element analysis and found to be in good agreement; the discrepancies were found to be less than $0.1 \%$.

Figure 14, which is the high-frequency AC analogue of Fig. 5, shows the inductance as a function of $s / w$ for various ratios of $t / w$. When $s / w$ is large, the inductance shows logarithmic dependence on $s / w$ as predicted by the large separation models [1]. As the gap between the conductors $(s-t)$ tends to zero the inductance also tends to zero. For very small gaps, the inductance may be approximated by $\mu_{0}(s-t) / w$. However, the curves rapidly depart from this asymptote as the separation increases. This behaviour is illustrated in Fig. 15.

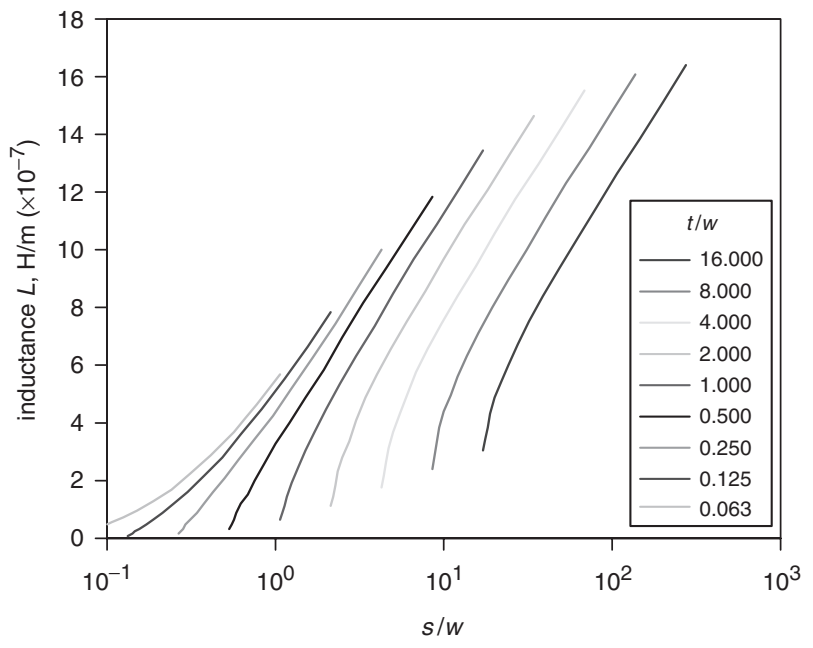

Fig. $14 L$ as a function of $s / w$ for various $t / w$

\section{Calculation of the losses}

The losses are obtained from the integral of the Poynting vector around the surface of the conductor which gives the power flow into the conductor, i. e.:

$$
\text { power flow }=\oiint(\boldsymbol{E} \times \boldsymbol{H}) . d \boldsymbol{S}
$$

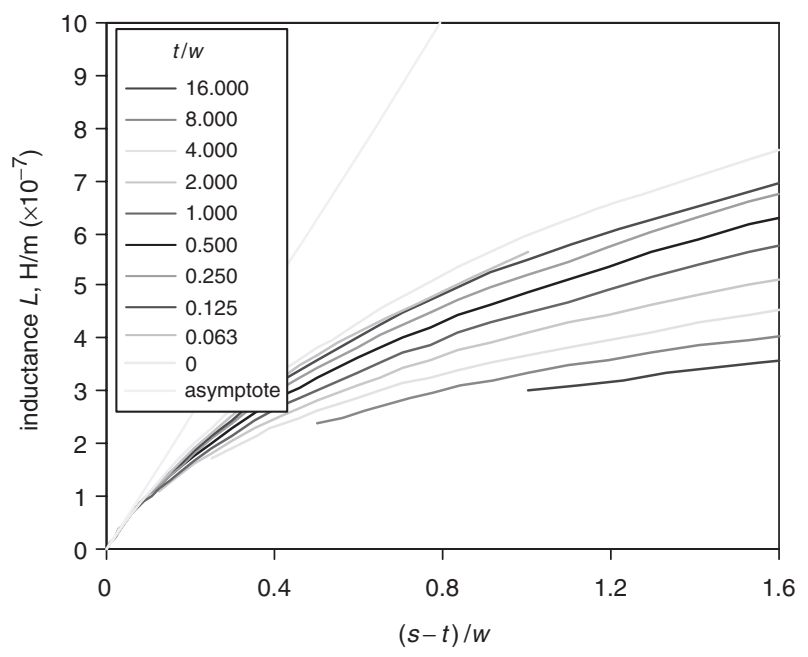

Fig. $15 L$ as a function of $(s-t) / w$ for various $t / w$

For the 2D case considered:

$$
H_{z}=0 \text { hence }(\boldsymbol{E} \times \boldsymbol{H}) \cdot \hat{\boldsymbol{n}}=E_{z} H_{\text {surf }}
$$

and

$$
R=\frac{\int\left(\oint E_{z} H_{\text {surf }} d s\right) d t}{\int I^{2} d t}
$$

where the time integrals are taken over a full period of the current waveform. If the skin depth is small in relation to the dimension of the rectangle a reasonable approximation can be obtained by assuming that $E_{z}$ and $H_{\text {surf }}$ are related by a $1 \mathrm{D}$ diffusion equation. If it is also assumed that the current waveform is sinusoidal, we find that:

$$
\frac{\int E_{z} H_{\text {surf }} d t}{\int H_{\text {surf }}^{2} d t}=\frac{1}{\sigma \Delta}
$$

and hence

$$
R=\frac{1}{\sigma \Delta} \oint \frac{H_{\text {surf }}^{2}}{I^{2}} d s
$$

The integral $\int H_{\text {surf }}^{2} d s$ in $s$-space may be converted into an integral along the real axis in $w$-space. If $\Omega$ denotes the scalar magnetic potential:

$$
H_{\text {surf }}=-\frac{d \Omega}{d s}
$$

and

$$
\begin{aligned}
\int H_{\text {surf }}^{2} d s & =\int\left|\frac{d \Omega}{d w} / \frac{d s}{d w}\right|^{2}\left|\frac{d s}{d w}\right| d w \\
& =\left|\frac{d \Omega}{d z}\right|^{2} \int\left|\left(\frac{d z}{d w}\right)^{2} / \frac{d s}{d w}\right| d w
\end{aligned}
$$

As before, values of $c, b_{2}, b_{1}$ and $a$ are computed numerically to produce the given geometry. Note that:

$$
\begin{aligned}
\frac{d \Omega}{d z} & =\left(\frac{I}{2|z(a)-z(c)|}\right) \text { and } \\
\frac{d z}{d w} & =\left(\frac{-1}{\sqrt{\left(w^{2}-a^{2}\right)\left(w^{2}-c^{2}\right)}}\right)
\end{aligned}
$$

from which it can be seen that:

$$
|z(a)-z(c)|=\frac{1}{a} \operatorname{elliptic} K\left(\sqrt{1-(c / a)^{2}}\right)
$$


Also

$$
\frac{d s}{d w}=\sqrt{\frac{\left(w^{2}-b_{1}^{2}\right)\left(w^{2}-b_{2}^{2}\right)}{\left(w^{2}-a^{2}\right)\left(w^{2}-c^{2}\right)}}
$$

Therefore

$\left(\frac{d z}{d w}\right)^{2} / \frac{d s}{d w}=\left(\frac{1}{\sqrt{\left(w^{2}-a^{2}\right)\left(w^{2}-c^{2}\right)\left(w^{2}-b_{1}^{2}\right)\left(w^{2}-b_{2}^{2}\right)}}\right)$

Hence, the losses are given by:

$$
\frac{1}{\sigma \Delta} \int_{\text {surf }}\left|H_{\text {surf }}^{2}\right| d s=\frac{1}{\sigma \Delta}\left|\frac{d \Omega}{d z}\right|^{2} \int_{c}^{a}\left|\left(\frac{d z}{d w}\right)^{2} / \frac{d s}{d w}\right| d w
$$

where the integrand on the right-hand side is given by (6). The integration in $w$-space is performed along a contour representing the surface of the conductor, i.e. between $c$ and $a$. The integral is evaluated in three pieces using the technique described in Section 5 to remove the singularities.

In the case where the width is large compared to the separation, the ratio $a / c$ is very large. This may cause substantial numerical errors in the evaluation of the integrals. An alternative geometry that avoids these inaccuracies is given in Fig. 16. A rectangular region between the conductors is left untransformed; hence, allowance must be made for the current and the associated losses in this region. If this method is used, it is important that $d$ is sufficiently large that the field at the interface may be considered uniform.

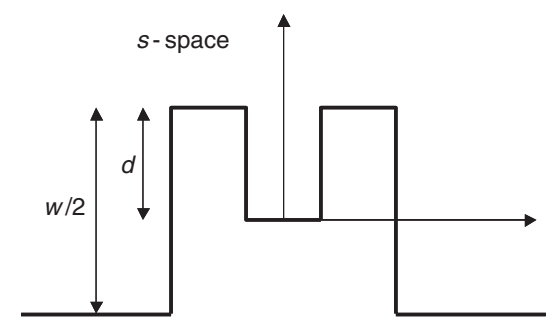

Fig. 16 A simpler type of geometry

Figure 17 shows $w \sigma \Delta R$ as a function of $s / w$. These results were obtained using the above semi-analytical method. When $s / w \rightarrow 0, H_{\text {surf }} \rightarrow I / w$ and so $\int H_{\text {surf }}^{2} d l / I^{2} \rightarrow 1 / w$. When $s / w \rightarrow \infty, \int H_{\text {surf }}^{2} d l / I^{2}$ tends to the value for an isolated strip with the same dimensions.

These results were compared against those obtained using finite element analysis. Initially, the finite element package was used to integrate $H^{2}$ around the surface of the conductor. In most cases, this yielded a result within $2.5 \%$ of the value obtained using the above method. However, in a few cases, the discrepancies were substantially larger.

To confirm that the largest errors arise in the integration of $H^{2}$ around the conductor in the finite element model, an alternative method was developed to evaluate $\int H_{\text {surf }}^{2} d l$

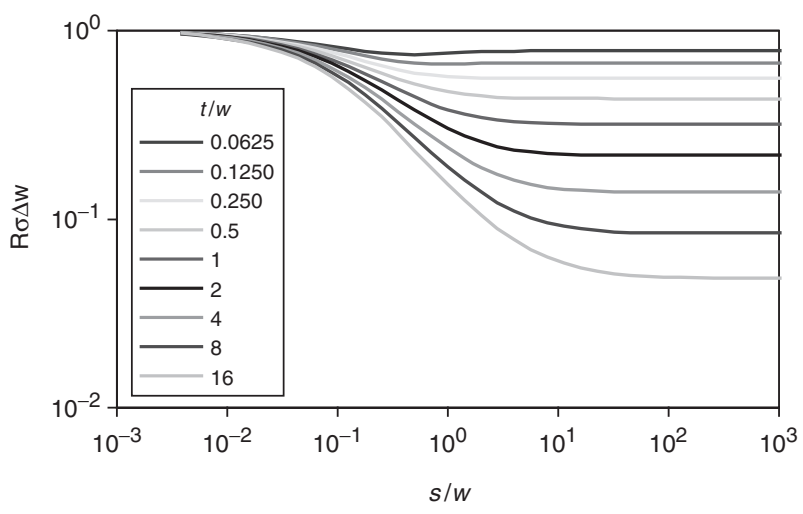

Fig. $17 w \sigma \Delta R$ as a function of $s / w$ for various ratios of $t / w$

within a short distance $r$ of the corner. This integral was evaluated using the values of vector potential on an arc at radius $r$ from the corner. This approach uses the conformal mapping $s=s_{0}+A w^{3 / 2}$ where $|A|=r$ and $s_{0}$ is the position of the corner. From Fourier analysis of the vector potential on the arc of radius $r$, a Taylor series is defined representing the potential in $w$-space. An analytical function was defined which gives $\int H_{\text {surf }}^{2} d l$ in terms of the coefficients of this Taylor series and the radius $r$. Using this method, the finite element results agree with the results obtained from the semi-analytical method to within $0.3 \%$.

\section{Conclusions}

For a pair of rectangular conductors it is possible to obtain an analytical expression for the DC inductance by direct integration. A good approximation to this result, which agrees to within $0.3 \%$, is obtained by expanding this expression in powers of $t / w$. An analytical expression for the DC inductance of three strips may be obtained using superposition of solutions.

The high-frequency AC resistance and inductance can be estimated using conformal mapping theory without requiring finite element software. The position of the poles and zeros that define the conformal mapping are obtained using numerical integration and optimisation functions. The inductance can be computed directly from these positions, while the resistance must be calculated by numerical

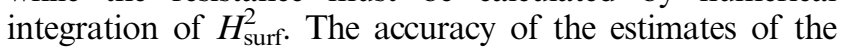
inductance and $\int H_{\text {surf }}^{2} d l$ obtained by this method was checked by comparison with results obtained using finite element analysis. Once measures were taken to control errors in $\int H_{\text {surf }}^{2} d l$ around the corners of the finite element model, the two methods agree to a high degree of accuracy.

\section{References}

1 Goddard, K.F., Roy, A.A., and Sykulski, J.K.: 'Inductance and resistance calculations for isolated conductors', IEE Proc., Sci., Meas. Technol., (in print)

2 Nehari, Z.: 'Conformal mappings' (McGraw-Hill, New York, 1952)

3 Grover, F.W.: 'Inductance calculations, working formulas and tables' (Dover, New York, 1962)

4 Abramovitz, M., and Stegun, I.A.: 'Handbook of mathematical functions' (Dover, New York, 1965) 